\title{
Avaliação do sistema integrado de controle parasitário em uma criação semi-intensiva de caprinos na região de Santa Catarina
}

\author{
[Evaluation of the integrated parasite control in a semi-intensive goat farming in the \\ region of Santa Catarina] \\ J. Hammerschmidt ${ }^{1}$, D. Bier ${ }^{1,3}$, F.S. Fortes ${ }^{1,3}$, P. Warzensaky ${ }^{2}$, A.M. Bainy ${ }^{2}$, \\ A.A.S. Macedo ${ }^{2}$, M.B. Molento ${ }^{3 *}$ \\ ${ }^{1}$ Aluno de pós-graduação - Universidade Federal do Paraná - UFPR - Curitiba, PR \\ ${ }^{2}$ Aluno de graduação - Universidade Federal do Paraná, UFPR - Curitiba, PR \\ ${ }^{3}$ Universidade Federal do Paraná, UFPR - Curitiba, PR
}

\begin{abstract}
RESUMO
O objetivo deste trabalho foi subsidiar um programa sustentável de controle parasitário em um rebanho caprino em São Francisco do Sul, SC, aplicando o Sistema Integrado de Controle Parasitário (SICOPA). Foram utilizados 63 caprinos, distribuídos em nove grupos para teste de eficácia de anti-helmínticos, exames de contagem de ovos por grama de fezes (OPG), coprocultura, grau Famacha, micro-hematócrito, escore corporal e contagem de larvas no pasto. A redução da OPG 15 dias pós-tratamento com closantel, albendazole, nitroxinil, levamisole, ivermectina+levamisole+albendazol, moxidectina, ivermectina, abamectina e sulfóxido de albendazol foi de $89,83,65,63,57,37,31,0$ e $0 \%$, respectivamente. A média de graus Famacha 1 e 2 no estudo foi de $81 \%$, e de graus 3, 4 e 5 foi de $19 \%$, e não se observou correlação com os valores do micro-hematócrito em razão da prevalência de Trichostrongylus sp. (92\%). A contagem de larvas infectantes $\left(\mathrm{L}_{3}\right)$ na pastagem apresentou valores abaixo de $1000 \mathrm{~L}_{3} / \mathrm{kg} / \mathrm{MS}$, predominando Trichostrongylus sp. Nenhuma das drogas testadas foi considerada eficaz, evidenciando resistência parasitária múltipla. A aplicação de ferramentas do SICOPA e a adoção de estratégias de manejo e nutrição adequados são fundamentais para estabelecer um programa sanitário sustentável.
\end{abstract}

Palavras-chave: anti-helmínticos, caprinos, parasitose, tratamento seletivo, SICOPA

\begin{abstract}
The objective of this study was to subsidize a sustainable parasite control program in a flock of goats in São Francisco do Sul, SC, Brazil, applying the integrated system for parasite control. Sixty three adult animals were used in nine groups to perform an anthelmintic efficacy test, faecal egg count (FEC), Famacha method, haematocrit, body condition score, coproculture, and the presence of larvae on pasture. Drug efficacy measured by FEC 15 days post-treatment with closantel, ivermectin + Levamisole + albendazole, albendazole, nitroxinyl, levamisol, abamectin, ivermectin, moxidectin and albendazole was 85, 57, 83, 65, 63, 31, 28, 24 and 0\%, respectively. The Famacha score 1 or 2 was given to 81\% and scores 3, 4 or 5 were given to 19\%, without significant correlation with haematocrit values since the predominant was Trichostrongylus sp. (92\%). The count of infective larvae levels on pasture revealed values below $1000 \mathrm{~L}_{3} / \mathrm{kg} / \mathrm{DM}$ predominantly Trichostrongylus sp. None of the tested drugs was effective, showing multiple parasitic resistance. The correct application of the SICOPA and adoption of management strategies and proper nutrition are essential to establish a sustainable health program.
\end{abstract}

Keywords: anthelmintics, goats, parasitosis, selective treatment

Recebido em 29 de abril de 2011

Aceito em 15 de maio de 2012

* Autor para correspondência (corresponding author)

E-mail: molento@ufpr.br 


\section{INTRODUÇÃO}

A caprinocultura é uma atividade amplamente explorada nos países tropicais, com o objetivo de produzir carne em diferentes condições ambientais. $\mathrm{O}$ interesse pela criação de caprinos vem aumentando significativamente devido à facilidade de manutenção dos animais em rebanhos locais de pequeno e médio porte. Embora apresente altíssimo potencial para se destacar no mercado mundial de produtos de origem caprina, a participação do Brasil ainda está abaixo de 2\% (Food..., 2006). Uma das razões desse baixo desempenho pode ser o elevado índice de infecções parasitárias, que se sugere ser a maior causa de prejuízos e alta mortalidade para a produção de caprinos (Githigia et al., 2001). Os principais nematódeos envolvidos nestas infecções são Haemonchus contortus, Trichostrongylus sp., Strongyloides papillosus e Oesophagostomum colubianum, os quais são considerados de grande importância econômica para a exploração de caprinos (Papadopoulos et al., 2001).

Roberts e Swan (1982) relataram que a maioria dos animais de um mesmo rebanho pode apresentar baixo nível de infecção, com um número inferior a $20 \%$ dos animais contendo graus indesejáveis de parasitismo. Mesmo assim, a infecção destes animais pode ser severa a ponto de causar perdas econômicas significativas. Isto significa que, quando se calculam as perdas produtivas assumindo a infecção como um efeito de distribuição homogênea no rebanho, podemse superestimar os dados, determinando, erroneamente, a projeção do prejuízo econômico final (Molento, 2004a).

O controle das nematodioses tem sido realizado, predominantemente, com o uso de antihelmínticos. No entanto, falhas têm sido relatadas, possivelmente devido ao fenômeno da resistência dos parasitos aos diferentes princípios ativos. Este fenômeno está amplamente difundido e preocupa a sustentabilidade das criações animais, sendo detectado quando a eficácia de uma droga falha em alcançar 95\% (Coles et al., 2006). O aparecimento da resistência parasitária é praticamente inevitável, e esta característica é transferida para as gerações de parasitos seguintes. O intervalo para que este fenômeno se inicie, todavia, dependerá da espécie do parasito, da pressão de seleção exercida pela droga e da frequência do tratamento nos animais (Conder e Campbell, 1995).

Em virtude do processo de resistência aos diferentes grupos químicos e da capacidade espoliativa dos helmintos, principalmente do gênero Haemonchus, faz-se necessária a busca de alternativas de controle. Neste contexto, insere-se o Sistema Integrado de Controle Parasitário (SICOPA), desenvolvido para as condições do produtor do Brasil (Molento, 2004b). O SICOPA consiste em um conjunto de estratégias para o tratamento parcial seletivo do rebanho, a fim de preservar parasitos com característica susceptível. Dessa forma, busca-se retardar a pressão de seleção dos helmintos e prolongar o tempo para o desenvolvimento de resistência, reduzindo, assim, os custos diretos de produção e o uso de drogas antiparasitárias (Torres-Acosta e Hoste, 2008; Kenyon et al., 2009).

O objetivo do presente trabalho foi avaliar as práticas de manejo propostas no SICOPA e a eficácia de compostos anti-helmínticos sobre parasitos gastrintestinais de caprinos, mantidos em uma criação semi-intensiva.

\section{MATERIAL E MÉTODOS}

O estudo foi realizado de abril a junho de 2006, em uma propriedade localizada no município de São Francisco do Sul, SC (26¹4'36”S, $\left.48^{\circ} 38^{\prime} 17^{\prime \prime} \mathrm{W}\right)$. O clima da região é descrito como tropical tipo $\mathrm{Cfa}$, com pluviosidade de 1400 a $2000 \mathrm{~mm}^{3}$, temperatura média anual de $21^{\circ} \mathrm{C}$ e umidade relativa do ar de $84 \%$. Inicialmente, foram coletados dados da propriedade por meio da aplicação de um questionário ao proprietário. Foram obtidas informações como manejo parasitário prévio, tipo de tratamento adotado, qualificação técnica local e assessoria profissional. A propriedade possuía 150 caprinos, 42 entre sete e oito meses de idade e 108 adultos, das raças Boer (PO) e Saanen (PO) e mestiços 3/4 e 7/8. Destes foram escolhidos 63 animais para serem incluídos no presente trabalho, com o teste de redução de contagem de ovos nas fezes (TRCOF). Todos os caprinos foram alocados em uma área com oito piquetes, em pastagem composta de Panicum sp., onde permaneciam por oito horas diárias e, no restante do período, eram mantidos em um capril elevado 
com piso ripado. Os animais receberam diariamente alimento concentrado e água $a d$ libitum.

Foram utilizados 63 caprinos adultos, naturalmente infectados, distribuídos em nove grupos ( $\mathrm{n}=7 /$ grupo). Para seleção e inclusão dos animais no experimento, foram utilizados os seguintes critérios: diagnóstico positivo obtido pelo número de ovos por grama de fezes; OPG e ausência de tratamento com qualquer droga antihelmíntica por um período mínimo de 90 dias. Foram utilizadas formulações comerciais dos seguintes compostos: abamectina, sulfóxido de albendazole, albendazol, closantel, associação albendazol + ivermectina + levamisole, ivermectina, levamisole, moxidectina e nitroxinil. Todos os animais foram devidamente pesados, e as doses administradas obedeceram à orientação do fabricante. Para avaliação da eficácia de cada anti-helmíntico, foram consideradas as contagens de OPG de cada grupo experimental no dia zero, e nos dias 15 e 30 após o tratamento. Dessa forma, cada grupo experimental, antes de receber o tratamento, desempenhou a função de controle, segundo a fórmula de Coles et al. (1992):

$\%$ eficácia $=$ média $\mathrm{OPG}$ do grupo controle média do OPG grupo tratado, dividido pela média do OPG do grupo controle e multiplicado por 100 .

As colheitas de fezes foram realizadas 15 dias antes da aplicação dos compostos antihelmínticos - dia -15 -, no dia do tratamento dia $0-$, e 15 e 30 dias pós-tratamento. As amostras foram colhidas diretamente da ampola retal de cada animal para determinação da OPG (Gordon e Whitlock, 1939). No dia 30, foram realizadas coproculturas diferentes para cada grupo de compostos anti-helmínticos testados, de acordo com a técnica adaptada de Roberts e O’Sullivan (1950). Um mínimo de 100 larvas infectantes $\left(\mathrm{L}_{3}\right)$ foram identificadas de acordo com Van Wyk et al. (2004).

Todos os animais foram examinados pelo método Famacha nos dias -15, 0, 15 e 30 após a vermifugação. O método consiste na avaliação a campo das diferentes tonalidades da conjuntiva ocular, com o objetivo de identificar clinicamente animais resistentes, resilientes e sensíveis às infecções por Haemonchus contortus, realizando-se o tratamento de forma seletiva. A coloração da conjuntiva pode variar de vermelho-rosada até branco-pálida, e sugere-se ser representada pelos números de 1 a 5 , sendo 1 a melhor coloração de conjuntiva e 5 o pior grau de anemia. O método possui um cartão- guia e a indicação de tratamento é baseada unicamente na coloração da conjuntiva, ou seja, animais com graus 3, 4 e 5 devem receber tratamento. (Van Wyk e Bath, 2002).

Nos dias 15 e 30 após tratamento, amostras de sangue de 25 animais escolhidos aleatoriamente foram colhidas diretamente da veia jugular em tubos BD Vacutainer com anticoagulante. O hematócrito foi realizado segundo o método do micro-hematócrito (Ferreira Neto et al., 1978).

A condição corporal dos caprinos foi aferida nos dias $-15,15$ e 30. O método de avaliação da condição corporal consistiu na palpação da região dorsal da coluna vertebral para a atribuição de valores de 1 a 5 , em que 1 representa animal caquético e 5 animal obeso (Russel et al., 1969).

A cada 15 dias foram colhidas amostras de pastagem de todos os piquetes ocupados pelos animais. A colheita foi realizada em sistema Z, em toda a extensão dos piquetes, sempre no período da manhã. As amostras foram processadas segundo a técnica de lavagem de pasto modificada por Molento (2004b). As larvas infectantes $\left(\mathrm{L}_{3}\right)$ foram identificadas (Van Wyk et al., 2004) para se determinar o número de $\mathrm{L}_{3}$ por quilograma de matéria seca de forragem $\left(\mathrm{L}_{3} / \mathrm{kg}\right.$ MS) em cada período do experimento.

A análise estatística para estabelecer correlações entre os dados obtidos com o grau Famacha, micro-hematócrito, OPG e escore da condição corporal foi realizada por meio de testes de hipóteses e de correlação de Pearson e Spearman. Os valores de correlação foram testados em um intervalo de confiança de $95 \% \quad(\mathrm{P}<0,05)$. A eficácia dos tratamentos relacionada com cada um dos gêneros de nematódeos foi calculada pelo programa estatístico RESO 2.0 (1989), o qual segue as instruções da Associação Mundial para o Avanço da Parasitologia Veterinária (WAAVP). 


\section{RESULTADOS E DISCUSSÃO}

O valor médio da OPG foi de 2400 , com valor mínimo de zero e máximo de 11100. A distribuição das contagem de ovos nas fezes, de acordo com os dias de coleta antes do tratamento - dia -15 - e após 15 e 30 dias, demonstrou um modelo de distribuição binomial negativa na relação parasito:hospedeiro, com distribuição agregada dos nematódeos antes e após o tratamento. Os resultados encontrados confirmam os já observados em ovinos por Barger (1985) e Amarante et al. (1998). Esses autores demonstraram que a infecção parasitária ocorre de forma individual e não de maneira uniforme, mesmo em animais da mesma raça e categoria de idade. Foi demonstrado que a maioria dos animais pode albergar poucos parasitos, enquanto um pequeno percentual está infectado com a maior proporção da população de parasitos (Molento et al., 2004).
No presente estudo, observou-se que em nenhum dos grupos de animais as drogas testadas apresentaram percentuais de redução de OPG superiores a 90\% (Tab. 1). Segundo critérios sugeridos pela Associação Mundial para o Avanço da Parasitologia Veterinária, WAAVP (Coles et al., 1992), dados de redução inferiores a $95 \%$ na contagem de OPG indicam a suspeita de resistência anti-helmíntica quando o intervalo de confiança for menor do que 90\%. Assim, considerando-se a contagem de ovos e os resultados da coprocultura observados no presente trabalho, há evidências de possível resistência dos helmintos identificados às bases avaliadas. Resultados semelhantes de resistência anti-helmíntica em nematoides de pequenos ruminantes foram observados em outros estudos na região Sul do Brasil (Thomaz-Soccol et al., 2004; Pereira et al., 2008), o que evidencia a necessidade de uso de estratégias alternativas para o controle dos parasitos em criações de animais.

Tabela 1. Percentual de redução da contagem de ovos por grama de fezes após tratamento com os diferentes fármacos testados em uma criação de caprinos, no âmbito da aplicação do Sistema Integrado de Controle Parasitário (SICOPA), no período de abril a junho de 2006, São Francisco do Sul, SC

\begin{tabular}{|c|c|c|}
\hline Anti-helmíntico & $\begin{array}{l}\text { Percentual de redução no } \\
\text { dia } 15\end{array}$ & $\begin{array}{c}\text { Percentual de redução } \\
\text { no dia } 30\end{array}$ \\
\hline Abamectina & Zero & 5 \\
\hline Albendazol & Zero & Zero \\
\hline Sulfóxido de albendazole & 83 & 54 \\
\hline Closantel & 89 & 51 \\
\hline Ivermectina+levamisole+albendazole & 57 & 67 \\
\hline Ivermectina & 31 & Zero \\
\hline Levamisole & 63 & 4 \\
\hline Moxidectina & 37 & Zero \\
\hline Nitroxinil & 65 & 26 \\
\hline
\end{tabular}

O closantel foi o antiparasitário com maior percentual de redução, com $89 \%$, mas mesmo assim ineficaz. O grupo das lactonas macrocíclicas, representado pelos antiparasitários abamectina, ivermectina e moxidectina, foi o que apresentou os piores resultados. Houve uma contradição de resultados entre dois produtos dos benzimidazois, representados pelo albendazol e pelo sulfóxido de albendazole, visto que o primeiro apresentou zero por cento e o segundo $83 \%$ de eficácia 15 dias após o tratamento. Foi possível observar que mesmo a combinação de princípios ativos com mecanismos de ação diferentes, que pode ser uma medida para melhorar a eficácia de cada droga isoladamente
(Food..., 2003), não apresentou resultados satisfatórios. Sugere-se que a baixa redução da carga parasitária observada após o tratamento pode ser oriunda do tratamento antiparasitário supressivo realizado por longos períodos. Além disso, é provável que algumas condições encontradas na propriedade, como a rápida rotação de princípios ativos e a ausência de diagnóstico parasitário anterior, tenham contribuído para o estabelecimento do quadro de resistência. Pode ter ocorrido a interação de fatores epidemiológicos, como a contaminação das pastagens e o clima favorável ao desenvolvimento dos nematódeos em sua forma infectante. A consideração destes fatores reforça 
a necessidade do monitoramento da eficácia das drogas, que é uma rotina pouco utilizada ou até mesmo inexistente, sendo que, na maioria dos casos, a resistência é determinada após a observação empírica da pouca eficácia da medicação utilizada (Molento, 2004a).

Foi observado que $100 \%$ das infecções helmínticas dos caprinos foram causadas por helmintos da superfamília Trichostrongyloidea.
A análise da coprocultura, realizada 30 dias após o tratamento, revelou o gênero predominante Trichostrongylus sp. (92\%), sendo observados Haemonchus sp., Strongyloides sp. e Oesophagostomum sp. nas proporções 7,8; 0,2 e $0,1 \%$, respectivamente (Tab. 2). Somente a ivermectina foi altamente ineficaz contra Haemonchus sp., revelando que Trichostrongylus sp. foi tolerante a todos os medicamentos testados.

Tabela 2. Percentual de larvas infectantes de helmintos parasitas encontrados 30 dias pós-tratamento de uma criação de caprinos, no âmbito da aplicação do Sistema Integrado de Controle Parasitário (SICOPA), no período de abril a junho de 2006, São Francisco do Sul, SC

\begin{tabular}{|c|c|c|c|c|}
\hline Grupo experimental & $\begin{array}{c}\text { Trichostrongylus } \\
\text { sp. }\end{array}$ & $\begin{array}{l}\text { Haemonchus } \\
\text { sp. }\end{array}$ & $\begin{array}{c}\text { Strongyloides } \\
\text { sp. }\end{array}$ & $\begin{array}{c}\text { Oesophagostomum } \\
\text { sp. }\end{array}$ \\
\hline Abamectina & $98 \%$ & $2 \%$ & Zero & Zero \\
\hline Sulfóxido de albendazole & $96 \%$ & $4 \%$ & Zero & Zero \\
\hline Albendazole & $90 \%$ & $10 \%$ & Zero & Zero \\
\hline Closantel & $96 \%$ & $4 \%$ & Zero & Zero \\
\hline Ivermectina+levamisole+albendazole & $100 \%$ & Zero & Zero & Zero \\
\hline Ivermectina & $60 \%$ & $40 \%$ & Zero & Zero \\
\hline Levamisole & $94 \%$ & $4 \%$ & $2 \%$ & Zero \\
\hline Moxidectina & $94 \%$ & $6 \%$ & Zero & Zero \\
\hline Nitroxinil & $99 \%$ & Zero & Zero & $1 \%$ \\
\hline
\end{tabular}

O baixo índice pluviométrico durante o presente estudo também poderia explicar a alta ocorrência de Trichostrongylus sp. Cavele et al. (2009) verificaram maior prevalência de infecção causada por $H$. contortus nos animais durante a época chuvosa e a predominância de Trichostrongylus spp., à medida que se aproximou a estação seca.

A quantidade média de $\mathrm{L}_{3}$ encontrada na pastagem está apresentada na Tab. 3.
Houve predominância de larvas do gênero Trichostrongylus sp., sendo as taxas mais elevadas registradas no início do tratamento dos animais. Os valores encontrados foram abaixo de $1000 \mathrm{~L}_{3} / \mathrm{kg} \mathrm{MS}$. Isso pode ser devido ao déficit pluviométrico acentuado que antecedeu o experimento, pois a redução da umidade pode dessecar as larvas infectantes e promover a inativação dos ovos (Cunha et al., 2000).

Tabela 3. Total da contagem de larvas de helmintos recuperados após técnica de lavagem de pasto em área naturalmente infectada com helmintos parasitas de caprinos, no âmbito da aplicação do Sistema Integrado de Controle Parasitário (SICOPA), no período de abril a junho de 2006, São Francisco do Sul, SC

\begin{tabular}{lcccc}
\hline & Larva & \multicolumn{3}{c}{ Dias } \\
\cline { 2 - 5 } & -15 & 0 & 15 & 30 \\
\hline Trichostrongylus sp. & 0 & 269 & 83 & 81 \\
Oesophagostomun sp. & 87 & 74 & 33 & 52 \\
Haemonchus sp. & 0 & 16 & 33 & 8 \\
Cooperia sp. & 18 & 0 & 0 & 0 \\
\hline
\end{tabular}

Os percentuais de avaliação clínica utilizando o método Famacha nos dias -15, 0, e nos dias 15 e 30 após tratamento estão demonstrados na Fig. 1. Durante todo o período experimental, houve predominância dos graus Famacha 1 e $2(81 \%)$ sobre os graus 3, 4 e 5 (19\%). De acordo com essa classificação, seriam vermifugados somente os animais compatíveis com os graus 4 e 5 e, em 
alguns casos, com o grau 3 (Malan et al., 2001). Assim, o tratamento seletivo possibilita a permanência de uma população parasitária em refugia, livre do tratamento anti-helmíntico e da pressão de seleção para resistência (Van Wyk e Bath, 2002). Molento et al. (2004) observaram ausência de anemia em animais com contagem de OPG acima de 1500 que, provavelmente, seriam considerados animais resilientes, ou seja, aqueles indivíduos capazes de suportar altas cargas parasitárias sem comprometimento clínico. No presente estudo, alguns animais também apresentaram altos valores de OPG com grau Famacha entre 1 e 2, sem sinais de anemia, sugerindo a capacidade de alguns animais tolerantes-resistentes e/ou resilientes de suportar alta carga parasitária sem a necessidade de tratamento.

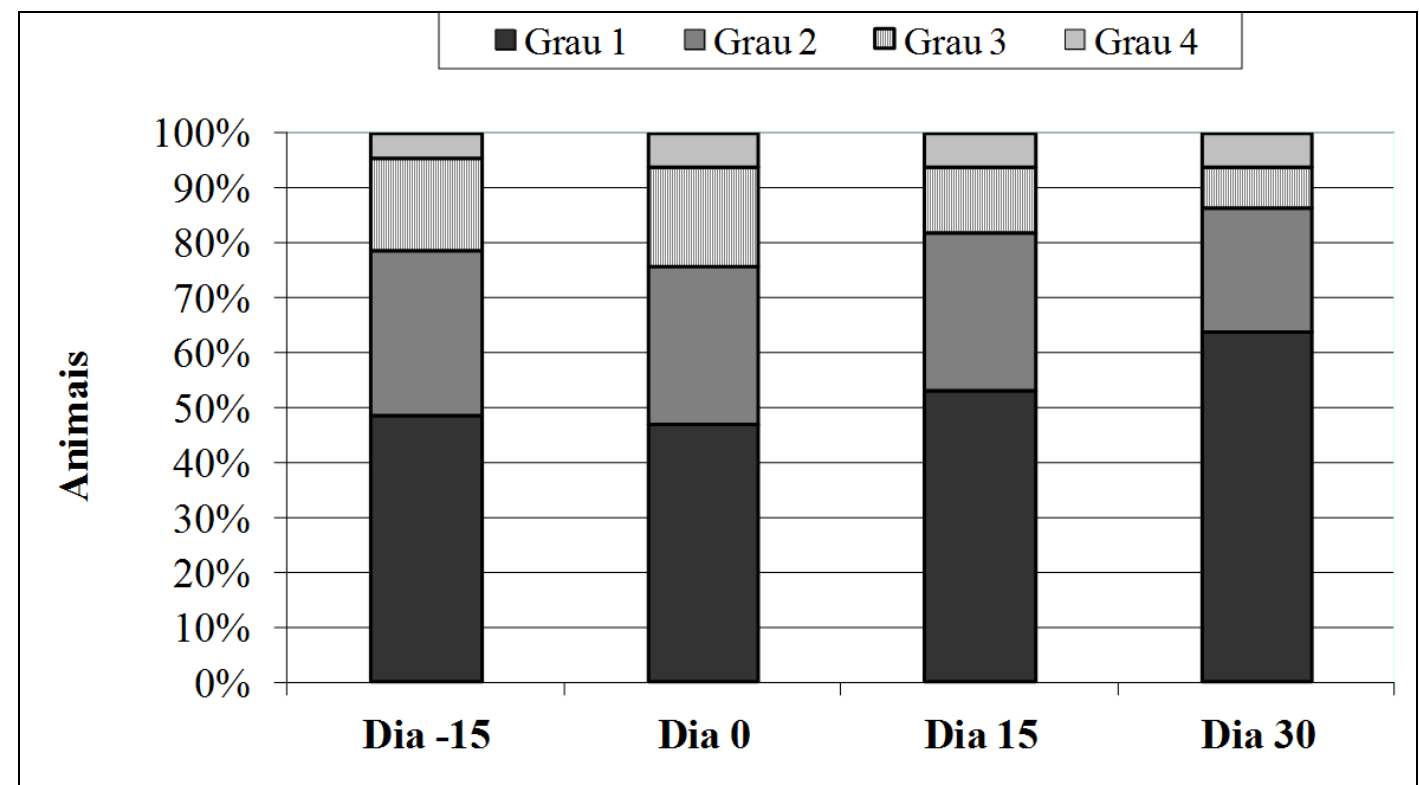

Figura 1. Percentuais de graus Famacha encontrados em uma criação de caprinos antes (dias -15, 0) e após (dias 15 e 30) tratamento anti-helmíntico, no âmbito da aplicação do Sistema Integrado de Controle Parasitário (SICOPA), no período de abril a junho de 2006, São Francisco do Sul, SC.

A avaliação média do escore corporal mantevese constante em 2,25 durante todo o experimento, sendo considerado abaixo do recomendado. O manejo nutricional inadequado empregado no período anterior ao experimento, a alta infecção parasitária e o período periparto podem ter colaborado para a baixa condição corporal do rebanho. A má nutrição pode ter contribuído, ainda, para a queda da imunidade dos animais e consequente aumento do grau de infecção (Houdijk et al., 2009). Práticas inadequadas de manejo adotadas na propriedade, como baixa altura dos comedouros, falta de histórico e identificação dos animais, ausência de acompanhamento técnico qualificado e tratamentos supressivos indiscriminados, são fatores considerados determinantes ao agravamento da parasitose na propriedade estudada.
Os dados obtidos neste experimento de OPG, grau Famacha, micro-hematócrito - entre 11 e $35 \%$; média de $25 \%$ - e escore corporal não apresentaram correlação significativa, como observado na Tab. 4, provavelmente devido à debilidade nutricional dos animais e ao predomínio de Trichostrongylus sp., tendo em vista que o método Famacha é aplicável em infecções por Haemonchus sp. (Van Wyk, 1999). Outros estudos em rebanhos de caprinos com maior grau de infecção por Haemonchus sp. encontraram correlação significativa entre os resultados de Famacha e micro-hematócrito (Molento et al., 2004; Cavele et al., 2009), e entre OPG e micro-hematócrito (Costa et al., 2000; Ejlertson et al., 2006; Cavele et al., 2009). 
Tabela 4. Valor de $P^{*}$ e correlação entre os dados de Famacha (FMC), contagem de ovos por grama de fezes (OPG), escore de condição corporal (ECC) e hematócrito $(\mathrm{Ht})$ em uma criação de caprinos, no âmbito da aplicação do Sistema Integrado de Controle Parasitário (SICOPA), no período de abril a junho de 2006, São Francisco do Sul, SC

\begin{tabular}{ccccc} 
& FMC & OPG & ECC & Ht \\
\hline FMC & - & 0,0342 & $-0,1936$ & $-0,267$ \\
OPG & 0,778 & - & 0,01573 & $-0,1903$ \\
ECC & 0,103 & 0,897 & - & 0,12687 \\
Ht & 0,140 & 0,297 & 0,489 & - \\
\hline
\end{tabular}

* Evidência estatística de correlação $(\mathrm{P}<0,05)$.

\section{CONCLUSÕES}

Foi possível aplicar o programa SICOPA e estabelecer um projeto sustentável de desenvolvimento em longo prazo. Nenhuma das drogas testadas foi considerada eficaz, o que evidenciou um processo de resistência aos antiparasitários já instalados na propriedade. Foi determinado que, mesmo evidenciando um processo de resistência parasitária múltiplo já instalado na propriedade, a correta aplicação de ferramentas do SICOPA e a deteç̧ão de falhas de manejo e nutrição podem permitir que se estabeleça um projeto sustentável de controle parasitário. Com o SICOPA, busca-se reduzir os custos diretos de produção, diminuindo o uso de compostos químicos, aumentando sua vida útil e, assim, retardando o processo de resistência parasitária.

\section{REFERÊNCIAS}

AMARANTE, A.F.T.; BARBOSA, M.A. Comparison between pasture sampling and tracer lambs to evaluate contamination of sheep pastures by nematode infective larvae. Rev. Bras. Parasitol. Vet., v.7, p.95-99, 1998.

BARGER, I.A. The statistical distribution of trichostrongylid nematodes in grazing lambs. Int. J. Parasitol., v.15, p.645-649, 1985.

BORDIN, L. Algumas considerações sobre a resistência de nematodas gastrintestinais de ruminantes aos anti-helmínticos. Rev. Bras. Parasitol. Vet., v.13, p.80-81, 2004.

CAVELE, A.; ALMEIDA, M.A.O.; BARRETO, M.A. et al. Estudo comparativo do sistema Famacha entre caprinos e ovinos sob o mesmo manejo produtivo no sertão baiano. Cienc. Anim. Bras., v.10, p.690-694, 2009.
COLES, G.C.; BAUER, C.; BORGSTEEDE, F.H.M. et al. World Association for the Advancement of Veterinary Parasitology (WAAVP) methods for detection of anthelmintic resistance in nematodes of veterinary importance. Vet. Parasitol., v.44, p.35-44, 1992.

COLES G.C.; JACKSON, F.; POMROY, W.E. et al. The detection of anthelmintic resistance in nematodes of veterinary importance. Vet. Parasitol., v.136, p.167185, 2006.

CONDER, G.A.; CAMPBELL, W.C. Chemotherapy of nematode infections of veterinary importance, with special reference to drug resistance. Adv. Parasitol., v.35, p.1-83, 1995.

COSTA, C.A.F.; VIEIRA, L.S.; BERNE, M.E.A. $e t$ al. Variability of resistance in goats infected with Haemonchus contortus in Brazil. Vet. Parasitol., v.88, p.153-158, 2000.

CUNHA, E.A.; BUENO, M.S.; SANTOS, L.E. Produção ovina em pastagens. In: CONGRESSO NORDESTINO DE PRODUÇÃO ANIMAL, 2., 2000; SIMPÓSIO NORDESTINO DE ALIMENTAÇÃO DE RUMINANTES, 8., 2000, Teresina. Anais... Teresina: SNPA, 2000. v.1, p.181-190.

EJLERTSEN, M.; GITHIGIA, S.M.; OTIENO, R.O. et al. Accuracy of an anaemia scoring chart applied on goats in sub-humid Kenya and its potential for control of Haemonchus contortus infections. Vet. Parasitol., v.141, p.291- 301, 2006.

FERREIRA NETO, J.M.; VIANA, E.S.; MAGALHÃES, L.M. Patologia clínica veterinária. Belo Horizonte: Rabelo e Brasil, 1978. 279p.

FOOD and agriculture organization - FAO. Resistencia a los antiparasitarios: Estado actual com énfasis en América Latina. Roma: Dirección de Producción y Salud Animal, 2003. 52p.

FOOD and agriculture organization of the united nations, 2006. FAOSTAT. Food and agriculture commodities. FAO/UN Report. 
GITHIGIA, S.M.; THAMSBORG, S.; MUNYUA, W.K. et al. Impact of gastrointestinal helminths on production in goats in Kenya. Small Rum. Res., v.42, p.21-29, 2001.

GORDON, H.M.; WHITLOCK, H.V. A new technique for counting nematode eggs in sheep faeces. J. Sci. Ind. Res., v.12, p.50-52, 1939.

HOUDIJK, J.G.; JACKSON, F.; KYRIAZAKIS, I. Nutritional sensitivity of resistance to Trichostrongylus colubriformis in lactating ewes. Vet. Parasitol., v.160, p.258-66, 2009.

KENYON, F.; GREER, A.W.; COLES, G.C. et al. The role of targeted selective treatments in the development of refugia-based approaches to the control of gastrointestinal nematodes of small ruminants. Vet. Parasitol., v.164, p.3-11, 2009.

MALAN, F.S.; VAN WYK, J.A.; WESSELS, C.D. Clinical evaluation of anaemia in sheep: early trials. Onderstepoort J. Vet. Res., v.68, p.165-174, 2001.

MOLENTO, M.B. Resistência de helmintos em ovinos e caprinos. Rev. Bras. Parasitol. Vet., v.13, p.82-87, 2004a.

MOLENTO, M.B. Sistema integrado de Controle parasitário, SICOPA. Revista CRMV-PR, v.3, p.23. 2007b.

MOLENTO, M.B.; TASCA, C.; GALLO, A. et al. Método FAMACHA@ como parâmetro clínico individual de infecção por Haemonchus contortus em pequenos ruminantes. Cienc. Rural, v.34, p.11391145, 2004.

PAPADOPOULOS, E.; HIMONAS, C.; COLES, G.C. Drought and flock isolation may enhance the development of anthelmintic resistance in nematodes. Vet. Parasitol., v.97, p.253-259, 2001.

PEREIRA, R.H.M.A.; AHID, S.M.M.; BEZERRA, A.C.D.S. et al. Diagnóstico da resistência dos nematoides gastrintestinais a anti-helmínticos em rebanhos caprino e ovino do RN. Acta Vet. Bras., v.2, p.16-19, 2008.
ROBERTS, I.H.; O'SULIVAN, P.J. Methods for egg counts and larval cultures for strongyles infesting the gastrointestinal tract of cattle. Aust. J. Agr. Res., v.1, p.99-102, 1950.

ROBERTS, J.; SWAN, R.A. Quantitative studies of ovine haemonchosis. The interpretation and diagnostic significance of the changes in serial egg counts of Haemonchus contortus in a sheep flock. Vet. Parasitol., v.9, p.211-216, 1982.

RUSSEL, A.J.F.; DONEY, J.M.; GUNN,R.G. Subjective assessment of body fat in live sheep. $J$. Agr. Scie., v.72, p.451-454, 1969.

THOMAZ-SOCCOL, V.; SOUZA, F.P.; SOTOMAIOR, C. et al. Resistance of gastrointestinal nematodes to anthelmintics in sheep (Ovis aries). Brazil. Arch. Biol. Technol., v.47, p.41-47, 2004.

TORRES-ACOSTA, J.F.J.; HOSTE, H. Alternative or improved methods to limit gastro-intestinal parasitism in grazing sheep and goats. Small Rum. Res., v.77, p.159-173, 2008.

VAN WYK, J.A.; BATH, G.F. The FAMACHA ${ }^{\odot}$ system for managing haemonchosis in sheep and goats by clinically identifying individual animals for treatment. Vet. Res., v.33, p.509-529, 2002.

VAN WYK, J.A.; CABARET, J.; MICHAEL, L.M. Morphological identification of nematode larvae of small ruminants and cattle simplified. Vet. Parasitol., v.119, p.277-306, 2004.

VAN WYK, J.A.; STENSON, M.O.; VAN DER MEERWE, J. et al. Anthelmintic resistance in South Africa: surveys indicate an extremely serious situation in sheep and goat farming. Onderstepoort J. Vet. Res., v.66, p.273-284, 1999. 